NASA Technical Memorandum 102078

AIAA-89-2906

\title{
Transient Flow Thrust Prediction for an Ejector Propulsion Concept
}

Colin K. Drummond

Lewis Research Center

Cleveland, Ohio

(NASA-TH-102078) TRAMSIEAT FLON THROST
PREDICTION FOR AN EJECTOB PROPOLSION CONCEPT
(NASA. Lewis Research Center) 10 pCSCL 21E

N89-24318

Unclas

G3/07 0210776

Prepared for the

25th Joint Propulsion Conference

cosponsored by the AIAA, ASME, SAE, and ASEE

Monterey, California, July 10-12, 1989 


\title{
Transient Flow Thrust Prediction for an Ejector Propulsion Concept
}

\author{
Colin K. Drummond* \\ National Aeronautics and Space Administration \\ Lewis Research Center \\ Cleveland, Ohio 44135
}

\begin{abstract} classic self-similar turbulent jet descriptions with a mixing region control volume analysis to predict transient effects in a new way. Details of the theoretical foundation, the solution algorithm, and sample calculations are given.

\begin{tabular}{ll} 
& \multicolumn{1}{c}{ Nomenclature } \\
$A$ & cross-sectional area \\
$b$ & jet half-width \\
$B$ & channel width \\
$C_{1}$ & coefficient defined by equation(24) \\
$K E$ & kinetic energy \\
$m$ & mass flowrate \\
$m_{c v}$ & control volume mass \\
$M_{1}$ & momentum flux \\
$M_{c v}$ & control volume momentum \\
$M_{1 P}$ & Mach number of primary nozzle flow \\
$P$ & pressure \\
$t$ & time \\
$v$ & velocity \\
$x$ & transverse coordinate \\
$z$ & streamwise coordinate \\
$Z_{i}$ & integral of defined function \\
$\rho$ & density \\
$\Phi$ & function defined by equation(4) \\
$\sigma$ & turbulent flow constant, equation(20) \\
$\xi$ & dimensionless transverse coordinate \\
& subscripts \\
$c v$ & control volume \\
$e$ & entrained stream \\
$m$ & primary stream centerline \\
$l s$ & secondary steam at station 1 \\
$l p$ & primary stream at station 1 \\
&
\end{tabular}

\section{Background}

A method for predicting transient thrust augmenting ejector characteristics is introduced. The analysis blends

Research on design methodologies for integrated aircraft and propulsion flight control systems requires accurate subsystem component simulations. In principle, these simulations must mimic steady-state and transient component effects. A NASA Lewis research program is currently underway to develop a "real-time" simulation, including system transients, for short take-off vertical landing (STOVL) aircraft. The thrust augmenting ejector shown in Fig. 1 is considered a potentially valuable propulsion subsystem element for the powered-lift aspect of STOVL aircraft. To explore ejector concepts further, the initial STOVL system simulation requires an ejector subsystem simulation.

For analysis, a thrust augmenting ejector is usually divided into a nozzle, inlet, diffuser and mixing regions. The ejector shown in Fig. 1 is a mechanically simple fluidic pump composed essentially of two components: (1) a "primary" jet nozzle issuing into (2) a shroud. This arrangement permits entrainment and acceleration of a secondary flow (within the shroud) by the primary jet. A diffuser section attached to the shroud allows control over the ejector discharge conditions.

An ejector simulation that includes ejector transients and with the potential to run real-time is not currently available; the motivation for the present work is the need to develop one. Of particular interest is the mathematical treatment of the ejector mixing region.

\section{Motivation for a New Modelling Approach}

Motivation for a new perspective on thrust augmenting ejector flows extends from the failure of existing mathematical models to meet current integrated flight / propulsion controls (IFPC) simulation requirements. A successful approach must satisfy three key constraints:

1. The formulation must handle unsteady flows and include a model for turbulent flow

2. The mathematical model must be predictive (not parametric) in nature,

3. The final system of equations must be amicable to a real-time simulation objective.

The first requirement points out the need for some type of characterization of turbulent mixing and entrainment phenomena in the mixing region; a description of these internal effects is essential for transient-type analyses. To meet the second requirement, only two data sets should be prescribed: (a) the primary jet "control-valve" setting (with associated thermodynamic data), and (b) free-stream

\footnotetext{
"Aerospace Engineer, Associate Member AIAA.
} 
at mospheric properties. From this, the secondary flow inlet conditions and the ejector thrust augmentation (as a function of time) are predicted. The third requirement emphasizes the ejector simulation must not become a bottleneck in the STOVL simulation (and thereby eliminating many traditional CFD methodologies in this application).

Constructing a mathematical model within these constraints is not a simple task; existing simulation approaches accommodating this scope were not found in the open literature. Traditional methods of analysis are described by Porter and Squires(1) and Addy and Dutton(2).

\section{Problem Solution Overview}

It is clear the IFPC ejector simulation task demands innovative approaches to unsteady turbulent flow treatment. Research at NASA Lewis has yielded a method for ejector analysis that represents a potential solution to the simulation problem. An empirically based model for the jet mixing turbulent interaction region is explored within the framework of a control volume analysis. This approach provides a rational foundation for the introduction of steady-flow data to "calibrate" an unsteady flow simulation. Preliminary performance predictions are intuitively reasonable, though verification of accuracy awaits availability of transient ejector performance data.

Here, a transient flow is a 'temporary' unsteady flow, associated with, for example, a change in ejector operation from one steady-state condition to another. Contrast this with oscillatory flows in which a periodic time-asymptotic flow character is exhibited. Ejectors utilizing pulsed primary nozzle flows are of the latter type. In the present simulation the focus on transient, not oscillatory, ejector phenomena descends from flight-critical aircraft flight control scenarios; an example would be transition to forward flight from vertical take-off.

Mixing Region Focus For the analysis of a given unsteady flow problem, it is quite convenient mathematically if a quasi-steady formulation can be assumed valid. This leads to the use of steady flow equations in an unsteady flow analysis; at each instant in time the flow is assumed to instantly respond to changes in the boundary conditions. The validity of such an assumption, however, depends on whether the characteristic time of the forcing function is the same order of magnitude as the relaxation time of the flow. In the present work only the inlet and diffuser benefit from the quasi-steady flow assumption; this directs our focus more appropriately on the control volume analysis of the mixing region. The notion here is that the physics of the mixing region are the cause of a situation that yields the pressure gradient effects in the inlet and diffuser.

Model Overview The mathematical framework of the present model is essentially a blend of three basic ideas. First, identify a finite number of control volumes (about five) of fixed size, partitioned only in the streamwise direction (the size of the control volumes are time independent). Translation of the Navier-Stokes equation into finite volume form provides three times as many algebraic equations as finite volumes. The robustness of the finite volume approximation minimizes the number of finite volumes needed; this is synonymous with reduced execution time.

Second, introduce the assumption that dimensionless self-similar velocity, temperature, and pressure distributions are applicable to the primary ejector flow and, furthermore, that the classic Abramovich(3) self-similar turbulent flow expressions are valid. This step provides the simplest turbulent model applicable to ejector flows. As a first approximation to meeting the primary and secondary jet mixing interface condition, the static pressure is assumed uniform in the transverse direction, but not in the streamwise direction. Characteristic profiles (see Fig. 2) provide a convenient link between the primary and secondary flows within the mixing region elements; Fig. 3 illustrates that there now results a very simple "virtual grid" on which to base the finite volume computations.

The third idea to introduce is that the kinetic energy gain of the secondary flow is expressible as a function of the kinetic energy loss of the primary flow. Contrast this with the traditional approach (see Korst and Chow ${ }^{4}$ ) of expressing the total kinetic energy of the secondary flow as a function of the total kinetic energy of the primary flow. A new kinetic energy function is needed since the traditional approach provides, by default, a quasi-steady flow result. In both cases an empirical constant must be introduced; in the traditional approach the constant derives from observation of normalized steady flow data -- in the present work the constant is determined by matching asymptotic thrust predictions for a given ejector performance window.

In summary, balances of mass, momentum, and energy are laminated on a simple virtual grid to provide the governing equations of the problem. Unlike many control volume analyses, the unsteady terms (time rate-of-change of, for instance, momentum of all particles inside the control volume) are not dropped in the present approach so we are forced to estimate field conditions in the ejector mixing region. In this work, the self-similar turbulent profiles approximate the velocity distribution within the control volume and the balance of energy divided into the (usual) thermal energy balance and a new function for mechanical energy.

\section{Formulation}

\section{Mixing Region Control-Yolume Equations}

A traditional one-dimensional flow approximation yields the following mass and momentum conservation laws:

Mass

$$
\frac{d}{d t}\left\{m_{c v}\right\}=\sum_{k} \dot{m}_{k}
$$

\section{Momentum}

$\frac{d}{d t} \int_{V} \rho \underline{v} d V=-\oint_{A} \rho \underline{v}(\underline{v} \cdot \underline{n}) d A-\oint_{A} \underline{n} \cdot(p \underline{I}) d A$ 
From a philosophical point of view, the presence of surface integrals (that in steady flow analyses yield sums of average quantities) reflects an increase in surface flux complexity, but allows for simplification of the volume integral representation. Control volume equations for energy and entropy balances are given by Thompson (5); for brevity of presentation the description of the finite volume method of this work will be restricted to equations(1) and (2). More details are given by Drummond(6).

\section{Turbulent Jet Approximation}

The present turbulent jet geometric approximation is shown in Fig. 2. Extending downstream from the mixing region inlet plane there exists a potential-core region characterized by a fairly uniform centerline velocity, with no transverse component. This is distinguished from the mixed-flow region where the centerline velocity decay arises from momentum transport to the entrained fluid.

Self-Similar Profiles In the characterization of an element of the mixing region, the velocity assumes a 2-D planar turbulent jet self-similar profile of Abramovich(3) for co-flowing jets:

$$
v=v_{e}(1-\Phi)+v_{m} \Phi=f(\Phi)
$$

where

$$
\Phi(\xi)=\left\{\begin{array}{cc}
\left(1-\xi^{1.5}\right)^{2}, 0 \leq \xi \leq 1 \\
0, & 1 \leq \xi \leq \xi
\end{array}\right\}
$$

and

$$
\begin{aligned}
\xi & =x / b \\
\xi & =B / b
\end{aligned}
$$

Inclusion of the core region requires a slight modification of these profiles; details are given in Abramovich(3).

Since there is a static pressure matching condition at the jet boundary, a uniform transverse pressure distribution is assumed; in the longitudinal (axial) direction a finite pressure gradient exists. This feature partially distinguishes free-jet and confined-jet analyses. As a first approximation, introduction of the uniform pressure profile into the ideal gas law suggests the transverse density gradient can also be uniform.

\section{Application to Conservation of Mass}

For the generic sub-region $k$, bounded by surfaces at $i$ and $j$, mass conservation yields

$$
\left(\frac{d m}{d t}\right)_{k}=\dot{m}_{i}-\dot{m}_{j}
$$

First, the mass flux is written

$$
m_{i}=\int_{A} \rho_{i} v_{i} d A=2 W \int_{0}^{\xi} \rho_{1} v_{i} d \xi
$$

From which integration over the self-similar profiles yields

$$
\begin{aligned}
\dot{m}_{t} & =2 W b_{i} \rho_{l}\left(0.45 v_{m}+0.55 v_{e}+(\xi-1) v_{e}\right)_{1} \\
& =2 W b_{l} Z_{1}
\end{aligned}
$$

The time-derivative term in the mass conservation equation is

$$
\left(\frac{d m}{d t}\right)_{k}=2 W B \Delta z\left(\frac{d \rho}{d t}\right)_{1}
$$

where the characteristic density for the finite volume is now approximated by the value of the density at station $j$ (in practice this is a good assumption as long as field variable gradients are "modest" in size). If the characteristic jet expansion width, $b$, also assumes its value at $j$, then substitution and re-arrangement of the continuity equation yields

$$
\left(\frac{d \rho}{d t}\right)_{,}=\frac{b, Z_{1, k}-b, Z_{1,1}}{B \Delta z}
$$

Computation of the jet half-width, $b$, derives from the momentum equation for incompressible flow applied to the first finite volume; a rectilinear jet expansion is assumed therefrom and compressible flow restored for subsequent calculations.

\section{Application to the Momentum Equation}

When the self-similar profiles are substituted into equation (2) and the algebraic dust settles, the jet centerline velocity derivative is

$$
\left(\frac{d v_{m}}{d t}\right)_{,}=\frac{\dot{M}_{k}}{2 W b, \Delta z}-\frac{v_{m}+v_{e} F_{1}}{\rho}\left(\frac{d \rho}{d t}\right)_{,}
$$

where

$$
\begin{aligned}
F_{1}= & \frac{\xi+0.45}{0.45} \\
\dot{M}_{k}= & 2 W b_{i} \rho_{i}\left(Z_{2.1}+\xi P_{1}\right) \\
& -2 W b_{,} \rho_{j}\left(Z_{2, j}+\xi P_{i}\right) \\
Z_{2}= & \int_{0}^{\xi} \rho_{k} v_{k}^{2} d \xi
\end{aligned}
$$

Note in equation (11) that the centerline velocity derivative is a function of the density derivative, equation (10).

Although application of the finite volume approximation has not been shown here for energy and entropy, the procedure is the same as described above. The result is a system of three equations describing the time derivatives of the jet centerline density, velocity, and temperature in terms of the state of the entrained flow. Integration of the system commences when the entrained flow state is defined.

\section{Entrained Velocity Augmentation}

Analysis of the primary and secondary flow interaction has not, to this point, been completed. By themselves, the self-similar profiles close the loop for steady-state flows, but not transient ones! This section provides an approximation for the turbulent flow kinetic energy exchange mechanism 
to characterize the influence of primary flow changes on the secondary flow. When the change in state of the secondary flow is established the corresponding secondary field variables can then be computed.

Secondary Stream Energy Change Computation of the gain in secondary flow kinetic energy due to mixing is given by

$$
\Delta K E=\int_{\zeta}^{\xi} \rho v\left(\frac{v^{2}}{2}-\frac{v_{*}^{2}}{2}\right) d \xi
$$

where $\zeta$ defines the jet boundary streamline (for which the primary mass flow through station $i$ is equal to primary mass flow through $j$ ). For the present discussion this dividing streamline position is assumed known; Korst and Chow(4) discuss the typical approach of analysis. Expanding the equation for the change in kinetic energy yields

$$
\Delta K E=W b \rho\left\{\int_{\zeta}^{1}\left(v^{3}-v v_{e}^{2}\right) d \xi+\int_{1}^{\xi}\left(v^{3}-v v_{e}^{2}\right) d \xi\right\}
$$

Substitution of the self-similar profiles into this expression and integrating the result provides

$$
\begin{aligned}
\Delta K E=N W b \rho\left\{v_{e}^{3}\right. & \left(H_{1}+H_{5}-F_{3}-F_{4}\right) \\
& +v_{m} v_{e}^{2}\left(H_{3}-F_{1}\right) \\
& \left.+v_{m}^{2} v_{e} H_{2}+v_{m}^{3} H_{4}\right\}
\end{aligned}
$$

where the detailed expressions for the integrals $H_{i}$ and $F_{i}$ are given by Drummond(6). It should be noted that in the present work the integrals are independent of time.

Primary Elow Energy Change Similar to the way in which the change in secondary flow kinetic energy was computed, the energy loss of the primary flow is given by

$$
\Delta K E=\int_{0}^{\zeta} \rho v\left(\frac{v^{2}}{2}-\frac{v_{m}^{2}}{2}\right) d \xi
$$

where the limits of integration reflect interest in the domain of the primary jet cross-section.

Evaluation of the integral at station $i$ yields the result

$$
\begin{aligned}
\Delta K E=W b \rho( & v_{e}^{3} H_{1}+v_{e} v_{m}^{2} H_{2} \\
& +v_{m} v_{e}^{2} H_{3}+v_{m}^{3} H_{4} \\
& \left.-v_{m}^{3} F_{1}-v_{e} v_{m}^{2} F_{3}\right)
\end{aligned}
$$

Again, reference(5) provides the expressions for the integrals implied in $F_{i}$ and $H_{i}$

Kinetic Energy Balance Computations for a specified steady-state condition show that the change in kinetic energy due to mixing is not the same for the secondary flow as it is for the primary. In fact, the gain in kinetic energy of the secondary flow is entirely due to the mixing process, while the mixing loss of the primary flow is only a fraction of its total loss. In balance, however, the total change of kinetic energy of the primary flow is greater than that of the secondary flow.

In the works of Korst and Chow(4) and Chow and Addy $(7)$ the relationship between the change in entrained flow kinetic energy and the total primary flow kinetic energy is given by

$$
E_{m}=\frac{\Delta K E_{1 s}}{\frac{1}{2} \rho_{1 p} v_{1 \rho}^{3}} \frac{\sigma}{z}
$$

where a value of 12 for $\sigma$ for turbulent flow traditionally provides a reasonable match between theory and experiment. A more accurate relationship includes the effect of the primary flow Mach number,

$$
\sigma=12\left(1+0.23 M_{1 \rho}\right)
$$

The important feature of this result is that the change in secondary flow kinetic energy has the functional form

$$
\Delta K E_{1 S}=F\left(K E_{1 P}, \frac{\sigma}{z}\right)
$$

The difficulty with the energy transfer function described above is that it provides (by default) a quasisteady flow approximation. It therefore cannot be used for the transient flow in its present form. To entertain local changes in primary-secondary kinetic energy in a way that does not burden the computational procedure, consider the modified function

$$
\Delta K E_{1 s}=f\left(\Delta K E_{1 P, m}, \Delta K E_{1 s, m}, \frac{\sigma}{z}\right)
$$

where the subscript $m$ denotes the change in kinetic energy due to mixing. The proposed form of this function is given by

$$
\Delta K E_{1 S}^{1 \cdot \Delta t}=\Delta K E_{1 S, m}^{1+\Delta t}+C_{1}\left(\frac{\sigma}{z}\right)^{2} \Delta K E_{1 P, m}^{1}
$$

This function for secondary flow $\mathrm{KE}$ enhancement arises from the assumption that local velocity gradients in the steady flow case are typically less than the gradients experienced in the transient mode. Here, the introduction of an engineering approximation also results in the introduction of an undetermined constant, $C_{1}$. The alternative is to establish $N$ computations of the kinetic energy exchange to coincide with the $N$ control volumes of the mixing region; the present method permits post-processing $\mathrm{KE}$ information at the completion of mixing region calculations. Numerical experimentation indicates

$$
0.1<C_{1}<0.5
$$

In summary, knowledge of the total change in secondary flow kinetic energy permits an update to the secondary flow state that corresponds with the dynamics of the primary flow. Once the velocity of the secondary flow has been extracted, the new inlet density and pressure are computed from an ideal flow inlet analysis. 


\section{Numerical Method}

Transformation of the control volume equations into finite-volume form provides a set of equations for the time-derivative of the field variables for each mixing region control-volume. These results participate in the solution as follows:

1. Define the computational grid (5 or so elements),

2. Initialize field variables by setting time derivatives to zero for mass, momentum, and energy, then solve for the associated (steady-state) field variable distribution,

3. Compute field variable time derivatives for each mixing region finite volume (use field variable values, as needed, from the previous time step),

4. Advance field variables forward in time explicitly using first order finite differences for the time-derivatives,

5. Update the entrained velocity,

6. Repeat steps 3-5 until desired time is reached.

Figure 4 summarizes this procedure.

\section{Results}

\section{Characteristic Test Case}

In the absence of data from transient flow ejector experiments (or even from modern multi-dimensional Navier-Stokes solvers), "verification" that the proposed ejector analysis can provide reasonable thrust predictions must be left to engineering judgement. Because of this, a "familiar" ejector forcing function must be used. In the present work the system response to a step-function input is not only a characteristic transient case study, but the scenario is also coincident with typical STOVL ejector application. For demonstration purposes the ejector system response to a step-change in primary nozzle flowrate from 18.7 to $21.85 \mathrm{lb} / \mathrm{m} / \mathrm{sec}$ is chosen because (a) experimental steady-state data at each of these operating points is available, and (b) the $17 \%$ change in primary flowrate is well beyond a "small"-perturbation examination (this exercises the system non-linearities).

\section{Calculation Results}

For the mixing region finite-volume length of $0.18 \mathrm{ft}$ and a characteristic mixing region velocity of $500 \mathrm{ft} / \mathrm{s}$, the characteristic time step for computations is approximately $0.4 \mathrm{~ms}$. To avoid infringing on this stability limit a computational time step of $0.1 \mathrm{~ms}$ was chosen; 100 time steps provided the necessary interval for examination of the step-function test case.

The empirical coefficient in the transient analysis (required for calibration of the primary-to-secondary kinetic energy exchange mechanism), was selected to match the asymptotic transient thrust prediction with the quasi-steady value at the new set point. Figure 5 illustrates the predicted ejector thrust profile for a coefficient $C_{1}$ of approximately 0.25 . It appears the 2 millisecond residence time of the flow (elapsed time for primary nozzle flow to reach the diffuser exit plane) is slightly less than the $\mathbf{3}$ millisecond interval for the thrust to reach a new maximum. Oscillations in thrust after that point appear to settle in about 5 milliseconds.

An unexpected feature of the thrust profile is the dip in thrust immediately following the step-change in primary nozzle efflux. Examination of the field variable profiles reveals this is not a numerical problem, but that the increase in static pressure associated with the instantaneous change in driving flow temporarily impedes the secondary flow. After a short period, the secondary flow kinetic energy builds (commensurate with the increase in primary flow energy) to overcome this effect, then continues in the intuitively expected manner.

\section{Remarks}

A distinctive second-order flavor is displayed by the predicted thrust profile; under a second-order assumption the ejector test case has approximately a 0.75 damping ratio and a natural frequency on the order of $300 \mathrm{~Hz}$. Although the results seem reasonable, it is necessary to conduct more extensive computational tests before conclusions about the order or linearity (about the perturbation) of the system can be made.

\section{Summary}

An ejector simulation method that includes ejector transients and with the potential to run real-time has been presented. The finite volume method permits rapid evaluation of the time dependence of field variables in a thrust augmenting ejector mixing region. Accuracy normally increases with an increase in the number of finite-volumes, though if the intent is to compute thrust, then quite reasonable results can be obtained with as few as five finite volumesb.

\section{References}

1. Porter,J. and Squyers,R. (1981), "A summary/overview of ejector augmentation theory and performance," USAF Technical Report No.R-91100-9CR-47.

2. Addy,A. and Dutton,J. (1974), "Ejector-diffuser theory and experiments," Report No.UILU-ENG-74-4009, University of Illinois at Urbana-Champaign.

3. Abramovich,G. (1963), The Theory of Turbulent Jets, MIT Press.

4. Korst,H. and Chow,W. (1966), "Non-isoenergetic turbulent jet mixing between two compressible streams at constant pressure," NASA-CR-419.

5. Thompson,P. (1972), Compressible Fluid Dynamics, New York: McGraw-Hill.

6. Drummond, C.K. (1988), "A Control-volume method of analysis of unsteady thrust augmenting ejector flows," NASA CR-182203.

7. Chow,W.L. and Addy,A.L. (1964), "Interaction between primary and secondary streams of supersonic ejector systems and performance characteristics," ALAA Joumal, V.2, No.4, pp.686-695. 


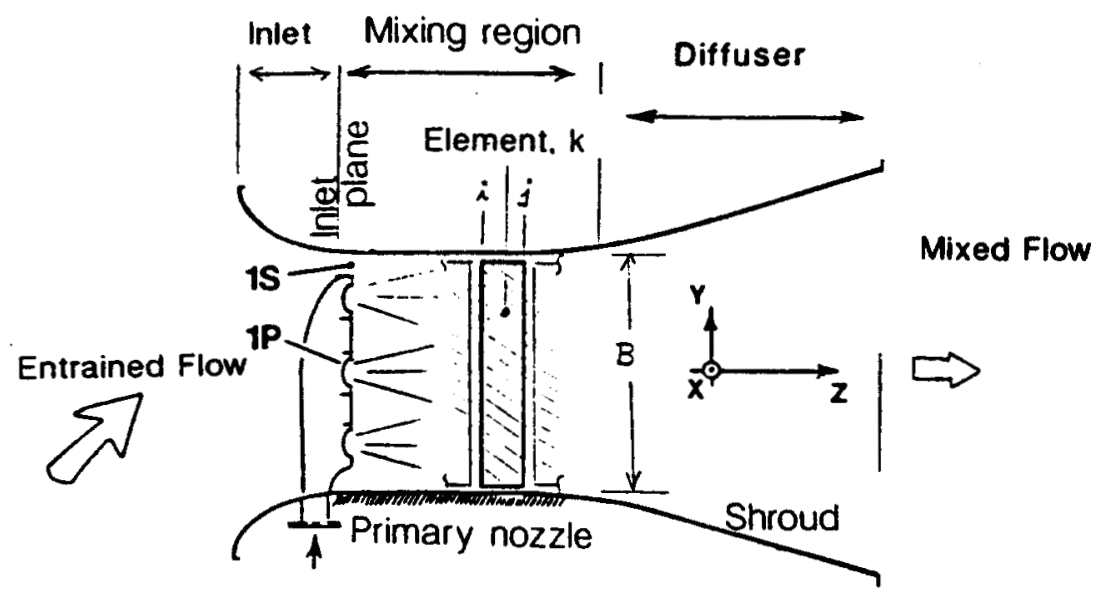

Fig. 1 Typical finite volume for ejector mixing region

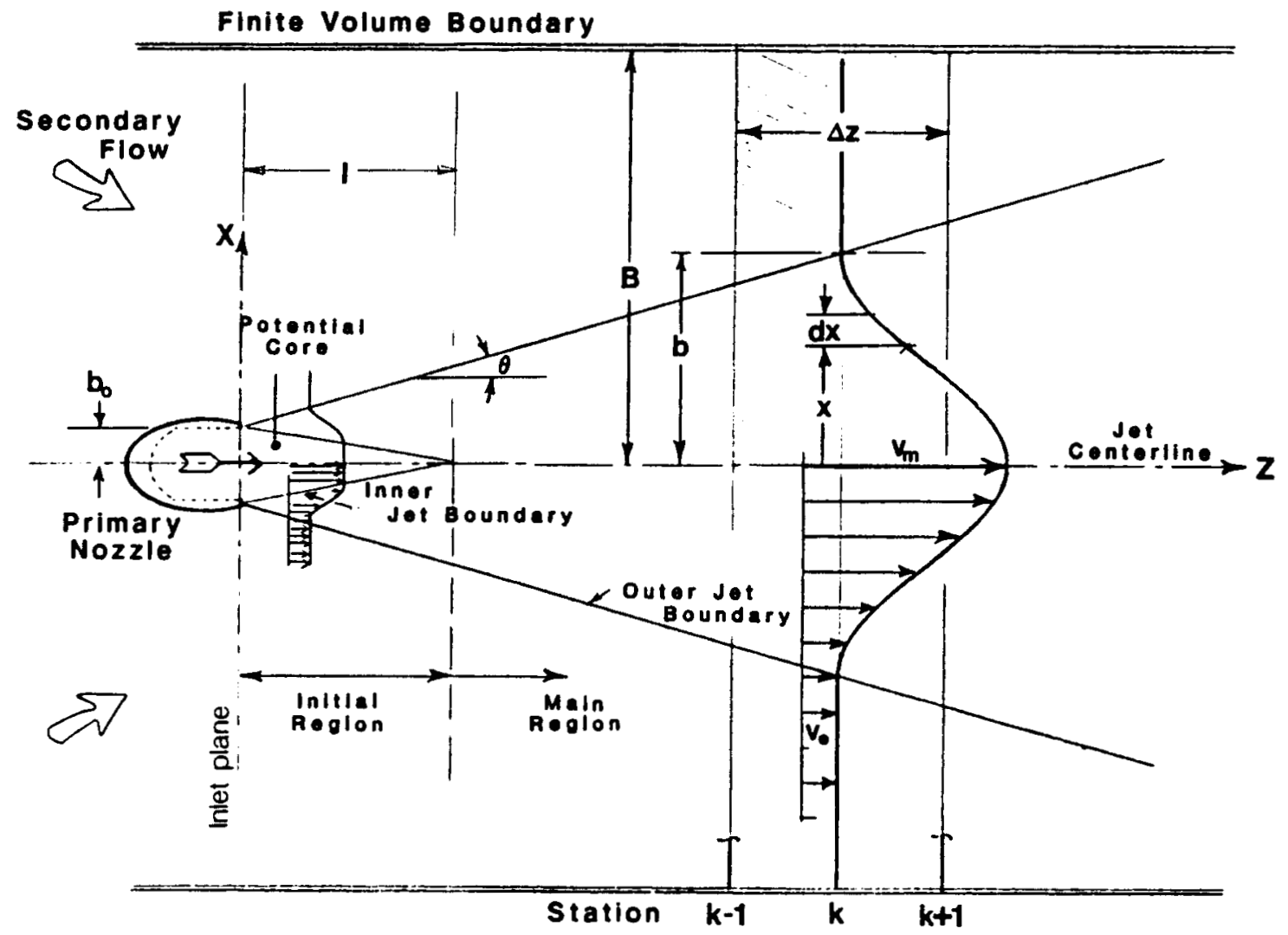

Fig. 2 Nomenclature for finite volume analysis 
Station

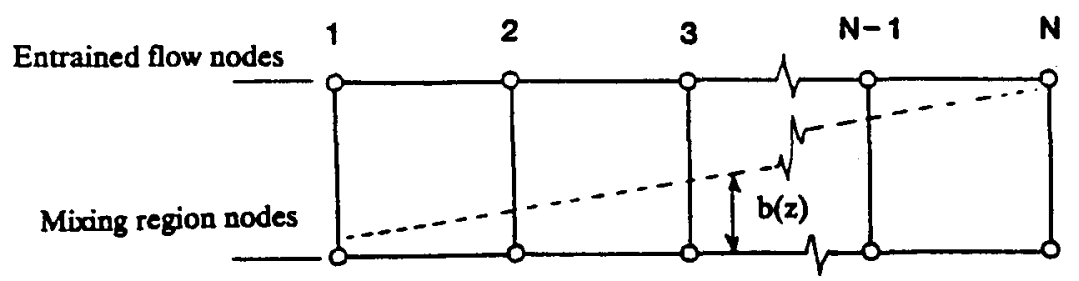

Fig. 3 Computational grid

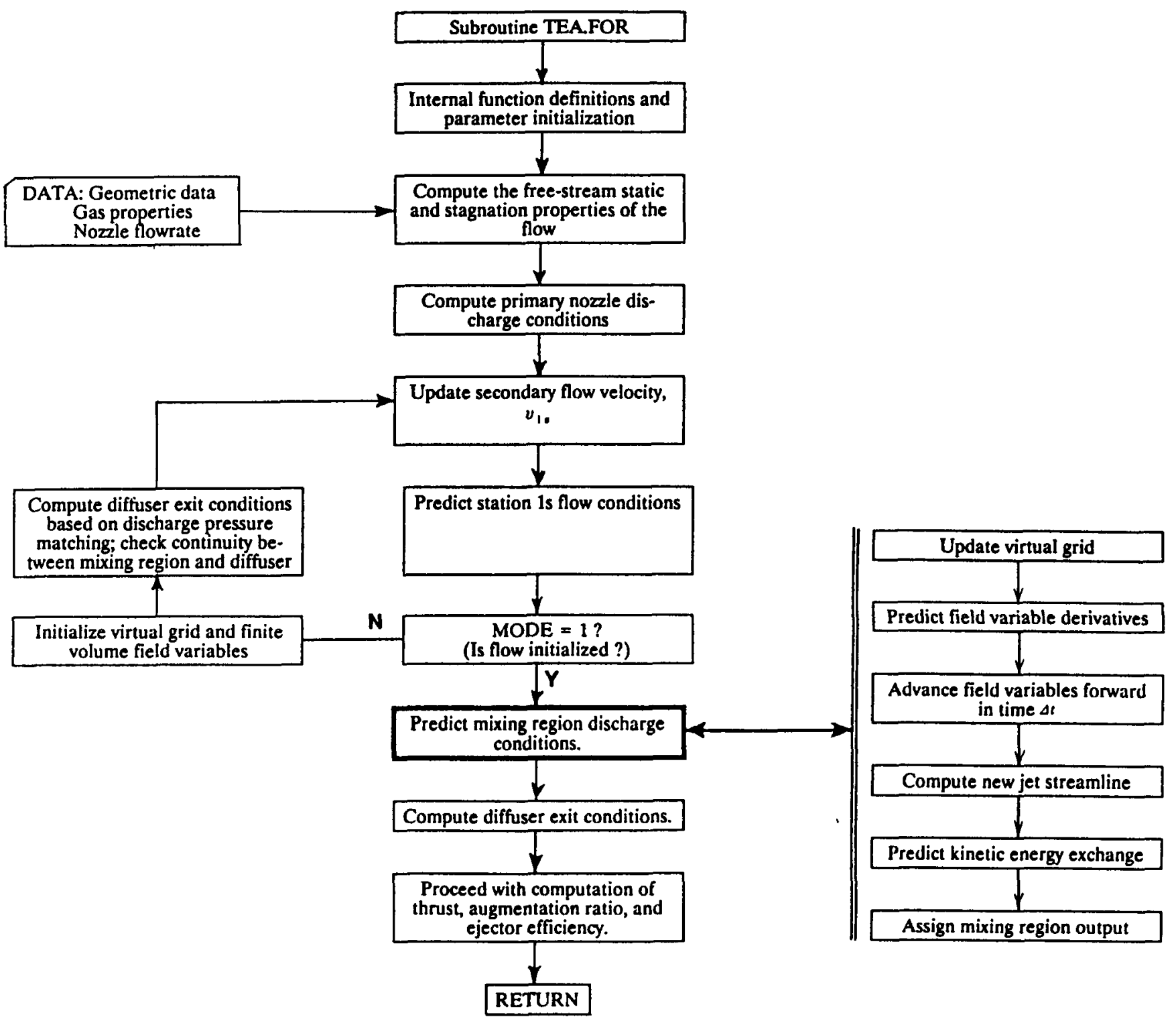

Fig. 4 Outline of solution Procedure 


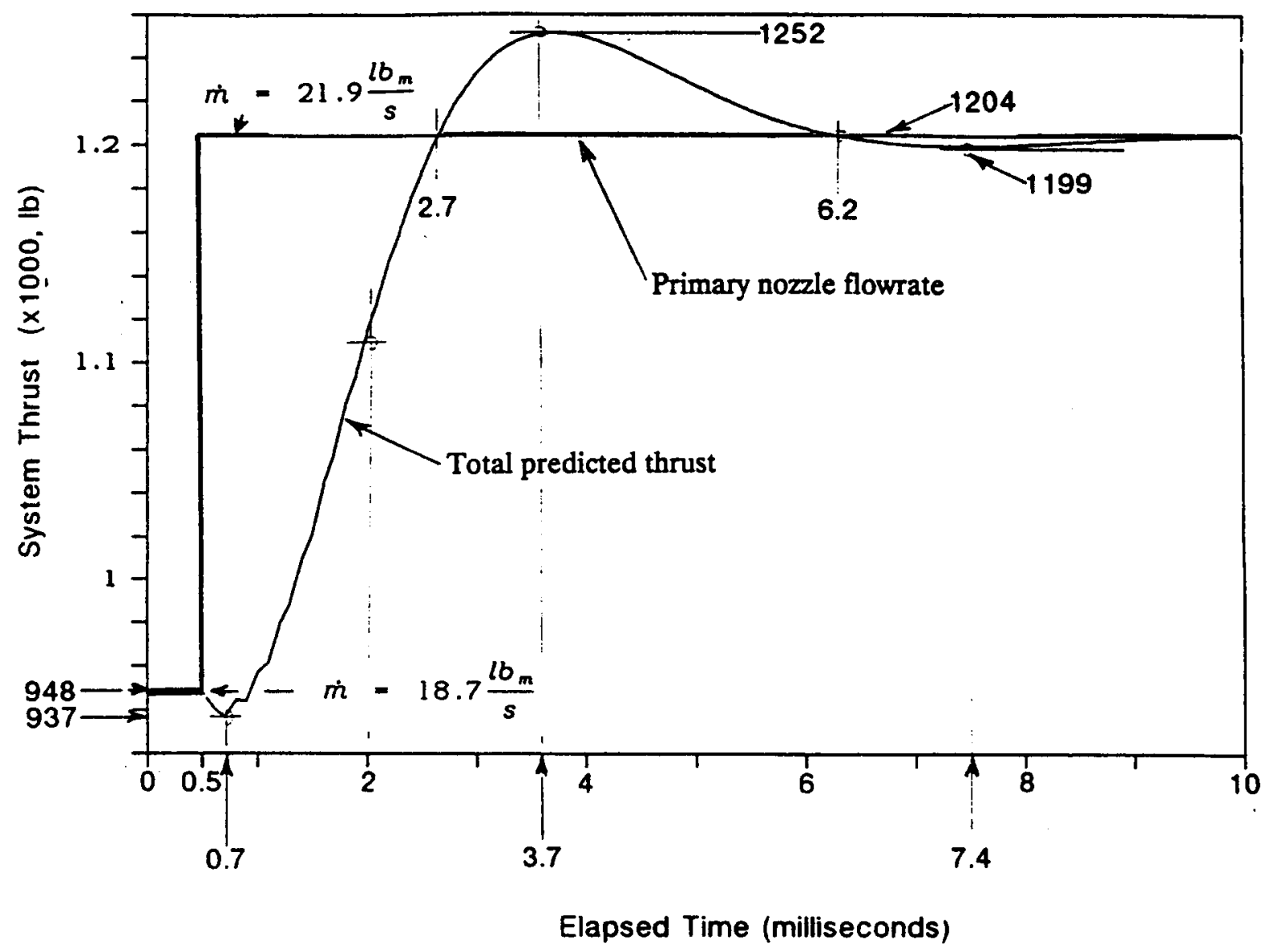

Fig. 5 Result from transient flow test case 


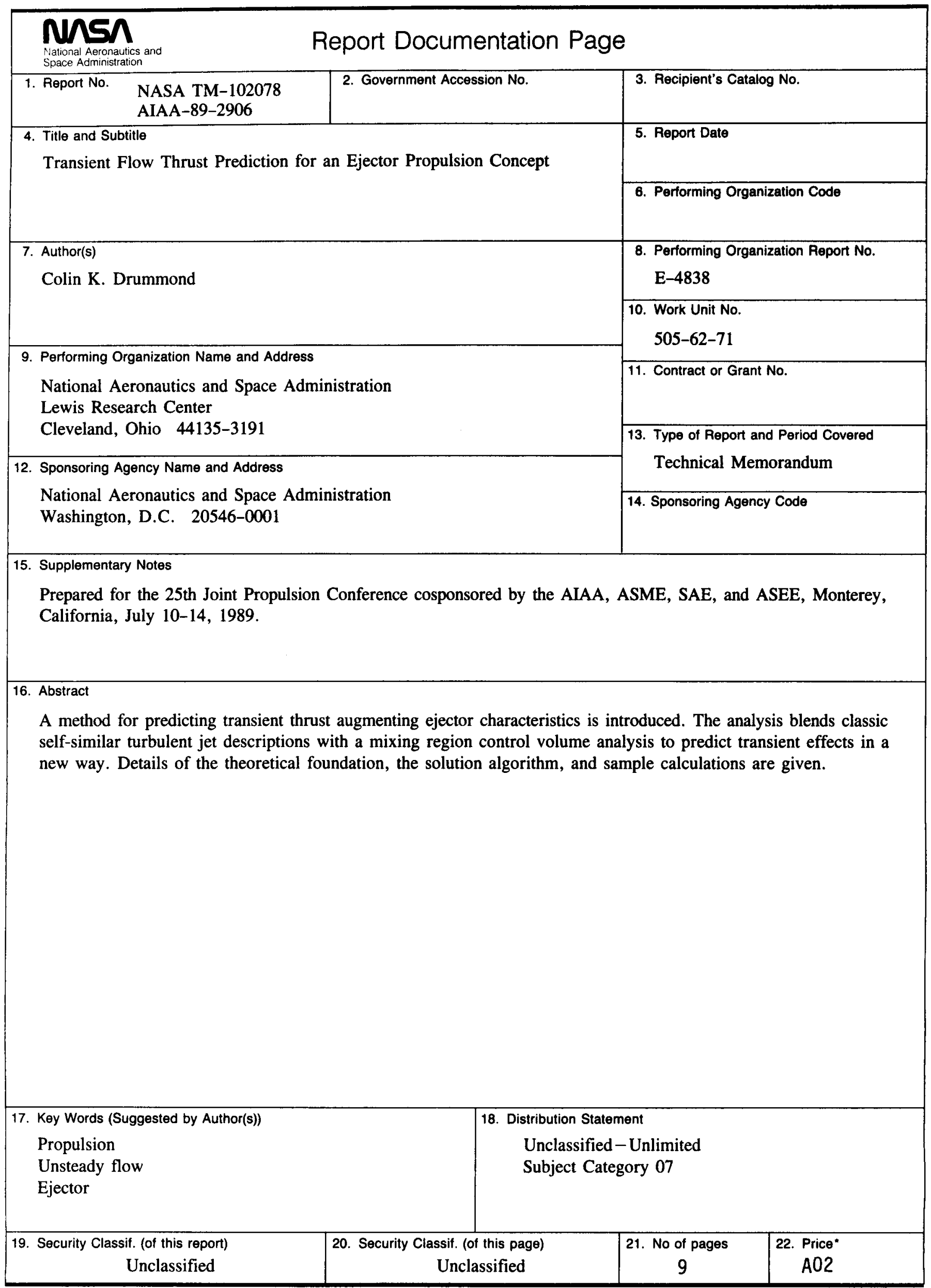

